\section{Relevance of Schneider's first-rank symptoms}

Peralta \& Cuesta (1999) accurately criticise the significant bias of prior studies, namely the inclusion of patients in whom schizophrenia was diagnosed using criteria that strongly rely upon first-rank symptoms. In their study the authors clearly showed the high prevalence of first-rank symptoms in schizophrenia and non-schizophrenic psychosis (diagnosed mainly by Feighner's criteria). They conclude that first-rank symptoms are not useful in differentiating schizophrenia from other psychotic disorders. This obviously seems correct, since Schneider (1959) himself never intended to differentiate schizophrenia from other psychoses (mainly schizophreniform disorder, schizoaffective disorder and atypical psychosis), as these disorders were part of the Schizophrener Formenkreis in Schneider's system. Therefore, we strongly disagree with Peralta \& Cuesta that the diagnostic relevance of first-rank symptoms should be to differentiate schizophrenia from other psychotic disorders.

Moreover, the authors conclude that until more evidence is available, first-rank symptoms "should not receive particular emphasis in the ICD-11 and DSM-V diagnostic criteria for schizophrenia". Peralta \& Cuesta omit a second possible conclusion: that the development of a nonschizophrenic psychosis (which, in fact, includes many of the diagnostic criteria of schizophrenia in any psychiatric classification) seems an artificial division of one clinical entity and this group should be considered as a schizophrenia subgroup in the ICD-11 and DSM-V.

Peralta, V. \& Cuesta, M. J. (1999) Diagnostic significance of Schneider's first-rank symptoms in schizophrenia. Comparative study between schizophrenic and non-schizophrenic psychotic disorders. British Journal of Psychiatry, 174, 243-248.

Schneider, K. (1959) Klinische Psychopathologie. New York/Stuttgart: Thieme Verlag.

F. Ortuño Department of Psychiatry and Medical Psychology, University of Navarra Medical School, Avda. Pio XII, 36, 31008 Pamplona, Spain

R. M. Bonelli Department of Neurology and Psychiatry, Hospital BHB Eggenberg, Berggasse 27, 802I Graz, Austria

Authors' reply: Drs Ortuño and Bonelli raise interesting questions regarding the relationship between our finding of lack of diagnostic value of first-rank symptoms for schizophrenia and Schneider's concept of schizophrenia. One of the major problems in interpreting the work of Schneider (1987) is that he described his concept of schizophrenia very succinctly. He mentioned clearly the pathognomonic value of firstrank symptoms for schizophrenia in the absence of organic illness (p. 65). However, the presence of first-rank symptoms, although sufficient, is not necessary for the diagnosis, which may be also done on the basis of 'second-rank' symptoms. Unfortunately, Schneider tells us nothing about how to diagnose the disorder on the basis of these symptoms, and as a consequence, beyond the presence of first-rank symptoms, the diagnosis of schizophrenia is only vaguely described. On the other hand, Schneider gives us a precise description of the boundaries of the concept when he states that "since in comparison with cyclothymia the diagnostic frame of schizophrenia is very broad and vague, we tend to include atypical cases within the varied clinical picture of schizophrenia" (Schneider, 1987, p. 8). It seems, therefore, that within the endogenous psychoses, for Schneider all that does not fit the diagnosis of cyclothymia is schizophrenia. His own data on the differential prevalence of endogenous psychosis (Schneider, 1987, p. 8) appear to support this assertion: $84 \%$ for schizophrenia, $15 \%$ for cyclothymia and $1 \%$ for mixed psychosis.

We agree that our study results, by showing that first-rank symptoms spread across the full spectrum of psychoses, do not necessarily contradict Schneider's schizophrenia concept, since DSM-III-R psychotic disorders and mood disorders with first-rank symptoms would correspond to Schneider's Schizophrener Formenkreis, which is very similar to the current notion of 'schizophrenia spectrum disorders'. Our statement that first-rank symptoms "should not receive particular emphasis in the ICD-11 and DSM-V diagnostic criteria for schizophrenia" refers to the way in which first-rank symptoms are used by these criteria, that is as symptoms having higher diagnostic prominence for schizophrenia than for non-schizophrenic psychoses. We agree that the division of psychotic disorders into schizophrenic and non-schizophrenic psychoses is an artificial one (our data regarding first-rank symptoms support this) as it is the radical separation between schizophrenia and manic-depressive illness made by Schneider. Notwithstanding, we do not agree with the contention that all psychotic disorders must be diagnosed as schizophrenia. If the schizophrenia concept makes any sense (at least in regard to historical and clinical reasons), it is to differentiate a subgroup of psychotic disorders with poor outcome; but precisely here is where the first-rank symptoms (and by extension, Schneider's schizophrenia concept) fail. Schneider held with Kraepelin's nosological distinction between schizophrenia and manic-depressive illness, and like him assumed a poorer prognosis for schizophrenia than for cyclothymia. He seems to think of first-rank symptoms when he mentions ". . . the task so important for clinical and social psychiatry, to search for symptoms, which from experience will permit predictions regarding the future course and outcome. This will after all be the question asked of us" (Schneider, 1925). The attribution of first-rank diagnostic value for schizophrenia to certain symptoms could be interpreted in the sense that their presence conveys poor outcome. However, the link between first-rank symptoms and poor outcome implicit in Schneider's writings is at odds with existing data indicating that first-rank symptoms are of no prognostic relevance (Mason et al, 1997). Therefore, given that (a) first-rank symptoms are present with a similar prevalence across the full spectrum of psychotic disorders, and (b) that first-rank symptoms are unrelated to the prognosis of schizophrenia, it seems fair to conclude that they are useless for the diagnosis of schizophrenia. In fact, our data support Crow's (1995) contention that they are not disease entities but continua of variation where schizophrenia and manicdepressive illness represent the extreme forms of the psychotic continuum.

Crow, T. J. (1995) A continuum of psychosis, one human gene and not much else: the case for homogeneity. Schizophrenia Research, 17, 135-145.

Mason, P., Harrison, G., Croudace, T., et al (1997) The predictive validity of a diagnosis of schizophrenia. A report from the International Study of Schizophrenia (ISOS), coordinated by the World Health Organization and the Department of Psychiatry, University of Nottingham.

Schneider, K. (1925) Wesen und Erfassung des Schizophrenen. Zeitschrift für die gesamte Neurology und Psychiatry, 99, 542-547.

- (1987) Klinische Psychopathologie (13th edn). Stuttgart: Georg Thieme Verlag.

V. Peralta, M. J. Cuesta Psychiatric Unit, Virgen del Camino Hospital, Irunlarrea 4, E-31008

Pamplona, Spain 